\title{
Evolução da morfologia e das propriedades mecânicas de blendas PA6/AES compatibilizadas com EPDM-MA
}

\author{
Evolution of morphology and mechanical properties of PA6/AES blends compatibilized with
} EPDM-MA

Evolución de la morfología y propiedades mecánicas de mezclas PA6/AES compatibles con EPDM-

\begin{abstract}
Resumo
Neste trabalho, a poliamida 6 (PA6) foi tenacificada com o terpolímero de acrilonitrila-EPDM-estireno (AES), utilizando o etileno-propileno-dieno enxertado com anidrido maleico (EPDM-MA), como agente compatibilizante. As blendas foram preparadas em uma extrusora de rosca dupla corrotacional e, posteriormente, moldadas por injeção. As propriedades de resistência ao impacto, resistência à tração e a morfologia foram avaliadas. Os resultados indicaram aumentos expressivos na resistência ao impacto das blendas PA6/AES/EPDM-MA, em comparação com a PA6 pura. Contudo, o módulo elástico e a resistência à tração sofreram decréscimos, devido ao aumento da flexibilidade das blendas desenvolvidas. A blenda PA6/AES compatibilizada com 15\% de EPDM-MA apresentou-se como um material supertenaz e dúctil na temperatura ambiente. As imagens obtidas por microscopia eletrônica de varredura (MEV) indicaram que o acréscimo no teor do compatibilizante EPDM-MA favoreceu à presença de partículas mais refinadas e bem aderidas na matriz de PA6, estabilizando assim a morfologia da blenda. Em geral, a formulação da blenda PA6/AES/EPDM-MA (60/25/15\%) apresentou potencial para aplicação de alto desempenho, com perspectivas para a produção de peças para suprir o mercado da indústria automobilista e da eletrônica.
\end{abstract}

Palavras-chave: Poliamida 6; AES; Tenacificação; Compatibilização; Propriedades mecânicas.

\begin{abstract}
In this work, polyamide 6 (PA6) was toughened with the terpolymer of acrylonitrile-EPDM-styrene (AES), using ethylene-propylene-diene grafted with maleic anhydride (EPDM-MA), as a compatibilizing agent. The blends were prepared in a co-rotational twin-screw extruder and subsequently injection molded. The properties of impact strength, tensile strength and morphology were evaluated. The results indicated expressive increases in the impact strength of the PA6/AES/EPDM-MA blends, compared to the neat PA6. However, the elastic modulus and tensile strength suffered decreases, due to the increased flexibility of the developed blends. The PA6/AES blend compatibilized with $15 \%$ of EPDM-MA presented itself as a supertough and ductile material at room temperature. The images obtained by scanning electron microscopy (SEM) indicated that the increase in the EPDM-MA compatibilizer content favored the presence of more refined and well-adhered particles in the PA6 matrix, thus stabilizing the blend's morphology. In general, the PA6/AES/EPDM-MA blend formulation (60/25/15\%) showed potential for high-performance application, with prospects for the production of parts to supply the automotive and electronics industry market.
\end{abstract}


Keywords: Polyamide 6; AES; Toughening; Compatibility; Mechanical properties.

\section{Resumen}

En este trabajo se endureció la poliamida 6 (PA6) con el terpolímero de acrilonitrilo-EPDM-estireno (AES), utilizando etileno-propileno-dieno injertado con anhídrido maleico (EPDM-MA), como agente compatibilizante. Las mezclas se prepararon en una extrusora de doble husillo co-rotacional y posteriormente se moldearon por inyección. Se evaluaron las propiedades de resistencia al impacto, resistencia a la tracción y morfología. Los resultados indicaron incrementos expresivos en la resistencia al impacto de las mezclas PA6/AES/EPDM-MA, en comparación con el PA6 puro. Sin embargo, el módulo elástico y la resistencia a la tracción sufrieron disminuciones, debido a la mayor flexibilidad de las mezclas desarrolladas. La mezcla PA6/AES compatible con un 15\% de EPDM-MA se presentó como un material super tenaz y dúctil a temperatura ambiente. Las imágenes obtenidas por microscopía electrónica de barrido (SEM) indicaron que el aumento en el contenido de compatibilizador EPDM-MA favoreció la presencia de partículas más refinadas y bien adheridas en la matriz de PA6, estabilizando así la morfología de la mezcla. En general, la formulación de la mezcla PA6/AES/EPDM-MA (60/25/15\%) mostró potencial para aplicaciones de alto rendimiento, con perspectivas de producción de piezas para abastecer el mercado de la industria automotriz y electrónica.

Palabras clave: Poliamida 6; AES; Endurecimiento; Compatibilidad; Propiedades mecánicas.

\section{Introdução}

O interesse no avanço do estudo das blendas poliméricas tem sido progressivo, visando uma alternativa para obtenção de novos materiais com propriedades desejadas e, consequentemente, atender à necessidade industrial (Luna et. al., 2015; Silva et al., 2016; La Mantia et al., 2017; Muthuraj et al., 2018; Luna et al., 2019). As blendas poliméricas podem ser obtidas por meio da mistura física de dois ou mais polímeros, utilizando equipamentos de processamento de polímeros commodities (Paul e Barlow, 1980; Luna et al., 2020a). Além disso, a produção de blendas apresenta um menor custo em comparação ao desenvolvimento de um novo polímero, o qual requer anos de pesquisas e reatores de polimerização. Em geral, a obtenção das blendas poliméricas tem como finalidade aperfeiçoar as propriedades mecânicas de termoplásticos de engenharia sensíveis ao entalhe, como a poliamida 6 (Nylon) (Hage e Pessan, 2001; Fernandes et al., 2012; Bezerra et al., 2014).

A poliamida 6 (PA6) faz parte dos primeiros termoplásticos comerciais de engenharia, tendo ligações do tipo amida (-CONH-) em sua estrutura molecular (Garcia, 1994; Luna et al., 2021). Possui amplas características como boa estabilidade dimensional, boa resistência química em meios orgânicos, boas propriedades mecânicas e térmicas (Bassani et. al., 2002). Todavia, uma limitação bastante conhecida da PA6 é sua baixa resistência ao impacto em temperaturas subambientes (Nogueira et al., 2021). Além disso, na presença de um entalhe, a PA6 apresenta um comportamento frágil, gerando limitações em aplicações que requeiram altas resistência ao impacto (Castro et al., 2016). Para aprimorar essa deficiência, em geral, adiciona-se uma segunda fase elastomérica, tendo em vista o processo de tenacificação a fim de favorecer uma melhoria dessa propriedade (He et al., 2017; Luna et al., 2015b). A tenacificação da PA6 para a produção de materiais com alta performance tem sido reportada na literatura, com blendas de PA6/PP/PP-g-MA (Sridhar \& Doddipatla, 2019), PP/ABS/SEBS-g-MA (Essabir et al., 2020), PA6/ABS/SMA (Oliveira, Larocca, \& Pessan, 2011), PA6/POE/POE-g-MA (Bo et al., 2021) e PA6/EPDM-MA (Luna et al., 2020b). Todavia, poucos trabalhos são relatados na literatura especializada de polímeros com o terpolímero acrilonitrila-EPDM-estireno (AES) para tenacificar a PA6, configurando, assim, uma lacuna tecnológica de grande relevância para ser explorada e aprofundada.

O AES é um modificador de impacto que apresenta potencial para tenacificar a poliamida 6. Esse modificador é constituído de EPDM, SAN livre e SAN grafitizado. O EPDM promove a compatibilidade entre as duas fases e, além disso, possui elevada resistência ao impacto e boa estabilidade térmica (Oliveira, 2014; Turchet e Felizbert, 2006). Em comparação com o ABS, o terpolímero de AES apresenta melhor resistência térmica e ambiental, maior resistência à oxidação da luz e a radiação ultravioleta, devido a substituição do polibutadieno por um elastômero com menor teor de insaturações (Carvalho, Gonçalves, \& Felisberti, 2010; Turchet \& Felisberti, 2006). Diante do potencial tecnológico do AES para tenacificar a PA6, 
houve algumas investigações na literatura.

Ghidoni et al. (1993) estudaram as propriedades mecânicas e a morfologia por dois métodos de blendas PA6/AES, utilizando o copolímero SMA e por meio da funcionalização do AES com anidrido maleico. Ao comparar os dois métodos, uma melhor propriedade mecânica foi observada usando a extrusão reativa. Ao comparar a adição de $10 \%$ e $24 \%$ de anidrido maleico no compatibilizante SMA, a reometria de torque indicou que $10 \%$ apresentou maior interação no sistema. Contudo, em termos de propriedades, a adição do SMA com $24 \%$ de MA apresentou melhores propriedades.

Bassani et al. (2005) analisaram a morfologia de blendas PA6/AES compatibilizadas com MMA-MA e MMA-GMA. A resistência à tração e a microscopia eletrônico de transmissão (MET) foram avaliadas. Os resultados indicaram que a incorporação direta do AES na matriz de PA6 não promoveu melhoria significativa na resistência ao impacto. Ao comparar os compatibilizantes, observou-se que o MMA-MA foi mais efetivo para compatibilizar a blenda PA6/AES, sugerindo um maior nível de interação entre as fases, gerando redução do tamanho das partículas da fase dispersa e, por consequência, uma alta resistência ao impacto.

Oliveira et al. (2017) estudaram blendas de PA6/AES compatibilizadas com MMA-MA. Os autores observaram que o teor de anidrido maleico presente no compatibilizante afeta as propriedades mecânicas. Os teores de 3 e $5 \%$ de MA no compatibilizante MMA-MA apresentaram características semelhantes nas propriedades mecânicas e na morfologia. Por outro lado, quando analisada a blenda com 10\% de MA, houve redução das propriedades mecânicas. Isso foi atribuído ao excesso de anidrido maleico que não reagiu.

Blendas binárias de PA6/AES necessitam de compatibilização para que haja uma maior interação entre as fases, contribuindo para promover uma dispersão adequada da fase elastomérica (Lipatov e Nesterov 1998). Com consequência, torna-se importante selecionar um compatibilizante adequado para interagir com a PA6 e o AES de forma simultânea, visando a obtenção de propriedades aprimoradas. Diante disso, o compatibilizante etileno-propileno-dieno enxertado com anidrido maleico (EPDM-MA) é um forte candidato para compatibilizar a blenda PA6/AES, haja vista que o anidrido maleico reage com os grupos terminais da PA6 e o EPDM apresenta miscibilidade com o componente elastomérico do AES. Em vista disso, a motivação para essa pesquisa é o desenvolvimento de blendas PA6/AES/EPDM-MA, uma vez que não se localizou na literatura investigações desse sistema polimérico.

A presente investigação teve como objetivo preparar blendas de PA6/AES com o compatibilizante com EPDM-MA, visando avaliar suas propriedades mecânicas e sua morfologia.

\section{Materiais e Métodos}

\subsection{Materiais}

A matriz polimérica foi a poliamida 6 (PA6), comercializada com o código B300®, densidade de $1,13 \mathrm{~g} / \mathrm{cm}^{3}$ e índice de fluidez de $2,9 \mathrm{~g} / 10 \mathrm{~min}\left(235^{\circ} \mathrm{C} / 2,16 \mathrm{~kg}\right)$, na forma de grânulos e fornecida pela Polyform.

O terpolímero acrilonitrila-EPDM-estireno (AES) foi utilizado como modificador de impacto, comercializado com o código Royaltuf $372 \mathrm{P} 20 ®$, densidade de $0,98 \mathrm{~g} / \mathrm{cm}^{3}$ e índice de fluidez de $20 \mathrm{~g} / 10 \min \left(265^{\circ} \mathrm{C} / 2,16 \mathrm{~kg}\right)$, na forma de grânulos e fornecida pela Addivant. Este material é constituído de EPDM, SAN e EPDM graftizado com SAN (EPDM-g SAN). Esta resina contém 50\% de EPDM e 50\% de SAN (valores fornecidos pelo fabricante) e 27\% de acrilonitrila no SAN livre.

$\mathrm{O}$ agente compatibilizante utilizado foi o etileno-propileno-dieno enxertado com anidrido maleico, comercializada com o código Royaltuf $498 \circledast$, contendo 0,8 a 1,2\% de anidrido maleico, na forma de grânulos e fornecida pela Addivant.

\subsection{Métodos}

A poliamida 6 foi submetida à secagem em estufa a vácuo com temperatura de $80^{\circ} \mathrm{C}$, por um período de 24 horas. $\mathrm{O}$ 
EPDM-MA e o AES foram secos em temperatura de $60^{\circ} \mathrm{C}$, sob vácuo, durante 24 horas. As composições desenvolvidas das blendas PA6/AES e PA6/AES/EPDM-MA estão apresentadas na Tabela 1, com as proporções massa (\%).

Tabela 1. Composições das blendas com as proporções em massa (\%).

\begin{tabular}{cccc}
\hline Nomenclatura & PA6 & AES & EPDM-MA \\
\hline PA6 & 100 & - & - \\
PA/AES & 60 & 40 & - \\
PA/AES/EPDM-MA & 60 & 35 & 5 \\
PA/AES/EPDM-MA & 60 & 32,5 & 7,5 \\
PA/AES/EPDM-MA & 60 & 30 & 10 \\
PA/AES/EPDM-MA & 60 & 27,5 & 12,5 \\
PA/AES/EPDM-MA & 60 & 25 & 15 \\
AES & 100 & - & - \\
\hline
\end{tabular}

Fonte: Dados da pesquisa.

As blendas PA6/AES e PA6/AES/EPDM-MA foram misturadas a seco e, subsequentemente, processadas em uma extrusora de rosca dupla corrotacional modular, modelo ZSK ( $\mathrm{D}=18 \mathrm{~mm}$ e L/D = 40), da Coperion Werner \& Pfleiderer. $\mathrm{O}$ processo de extrusão foi realizado com perfil de temperatura de $230^{\circ} \mathrm{C}$ em todas as zonas, vazão de alimentação de $2 \mathrm{~kg} / \mathrm{h}$ e rotação da rosca de $200 \mathrm{rpm}$. Para efeito comparativo, a PA6 foi processada nas mesmas condições das blendas. Após o processamento, o material foi granulado e seco em uma estufa com vácuo por $24 \mathrm{~h}$, sob temperatura de $80^{\circ} \mathrm{C}$.

As blendas obtidas por extrusão foram moldadas por injeção em uma injetora Arburg, Modelo Allrounder 207C Golden Edition, para obtenção de corpos de prova de impacto e tração, segundo as normas ASTM D256 e D638, respectivamente. As condições de moldagem por injeção foram: $230,240,240,240,245^{\circ} \mathrm{C}$, temperatura do molde de $50^{\circ} \mathrm{C}$, pressão de injeção de 1000 bar, pressão de recalque de 700 bar e tempo de resfriamento de 25 s. Após a moldagem por injeção, os corpos de prova foram armazenados em um dessecador por um período superior a 48h, até o momento das caracterizações.

\subsection{Caracterizações}

Os ensaios de tração foram realizados em corpos de prova injetados, segundo a norma ASTM D638, utilizando uma máquina de ensaios universal da marca EMIC DL 2000, com velocidade de carregamento de $50 \mathrm{~mm} / \mathrm{min}$ e célula de carga de $20 \mathrm{kN}$. Os testes foram conduzidos à temperatura ambiente e os resultados analisados com uma média de 10 corpos de prova.

O ensaio de resistência ao impacto Izod foi realizado em corpos de prova entalhados, segundo a norma ASTM D256, em um aparelho da marca Ceast modelo Resil 5,5 J, operando com martelo de 5,5 J, em temperatura ambiente. Os resultados foram analisados com uma média de 10 corpos de prova.

As análises por microscopia eletrônica de varredura (MEV) foram realizadas na superfície de fratura dos corpos de prova submetidos ao ensaio de impacto. Utilizou-se um microscópio eletrônico de varredura, VEGAN 3 TESCAN, a uma voltagem de $20 \mathrm{kV}$, sob alto vácuo. As superfícies de fratura das amostras foram recobertas com ouro.

\section{Resultados e Discussão}

\subsection{Comportamento mecânico sob tração}

Os resultados obtidos para as propriedades mecânicas de tração da PA6 e das blendas, com e sem compatibilizante EPDM-MA, estão sumarizados na Tabela 2. As Figuras 1, 2 e 3 mostram as tendências do módulo elástico, da resistência à tração e do alongamento na ruptura, respectivamente. A PA6 pura apresentou o mais alto módulo elástico entre todos os 
materiais, ou seja, a maior rigidez. A adição direta do AES reduziu em 52,7 \% o módulo elástico, em relação a PA6. Isso é devido ao comportamento elastomérico do AES, contribuindo para diminuir essa propriedade. Tal comportamento foi verificado na literatura com sistemas similares (Oliveira et al., 2017). Ao adicionar 5,0 e 7,5\% do compatibilizante EPDMMA, as variações no módulo elástico não são significativas, em relação a blenda binária. Nesse caso, essas blendas estão dentro da margem do erro experimental, ou seja, os módulos são equiparáveis entre si. Na faixa de composição de 10 a $15 \%$ de EDPM-MA, o módulo elástico das blendas PA6/AES/EPDM-MA reduziu de maneira mais evidente. Isso é atribuído ao acréscimo do componente elastomérico nas blendas, gerando maior flexibilidade e reduzindo a rigidez.

Tabela 2. Propriedades mecânicas para a poliamida 6 e as blendas.

\begin{tabular}{cccc}
\hline Blendas & $\begin{array}{c}\text { Módulo de } \\
\text { elasticidade } \\
(\mathbf{M P a})\end{array}$ & $\begin{array}{c}\text { Resistência à tração } \\
\text { (MPa) }\end{array}$ & $\begin{array}{c}\text { Alongamento na } \\
\text { ruptura }(\%)\end{array}$ \\
\hline PA6 & $2370,0 \pm 50$ & $58 \pm 4$ & $78,3 \pm 4$ \\
\hline PA6/AES & $1121,12 \pm 35$ & $25,2 \pm 0,9$ & $38 \pm 5$ \\
\hline PA6/AES/EPDM-MA (5\%) & $1095,8 \pm 40$ & $24,6 \pm 2$ & $58 \pm 5$ \\
\hline PA6/AES/EPDM-MA (7,5\%) & $1095,6 \pm 60$ & $25,1 \pm 1,5$ & $65 \pm 7$ \\
\hline PA6/AES/EPDM-MA (10\%) & $944,5 \pm 30$ & $23,6 \pm 1$ & $84 \pm 7$ \\
\hline PA6/AES/EPDM-MA (12,5\%) & $957,2 \pm 40$ & $23,9 \pm 1,1$ & $114,7 \pm 10$ \\
\hline PA6/AES/EPDM-MA (15\%) & $988,9 \pm 35$ & $25,3 \pm 0,8$ & \\
\hline
\end{tabular}

Fonte: Dados da Pesquisa.

Figura 1. Módulo elástico para a poliamida 6 e suas blendas.

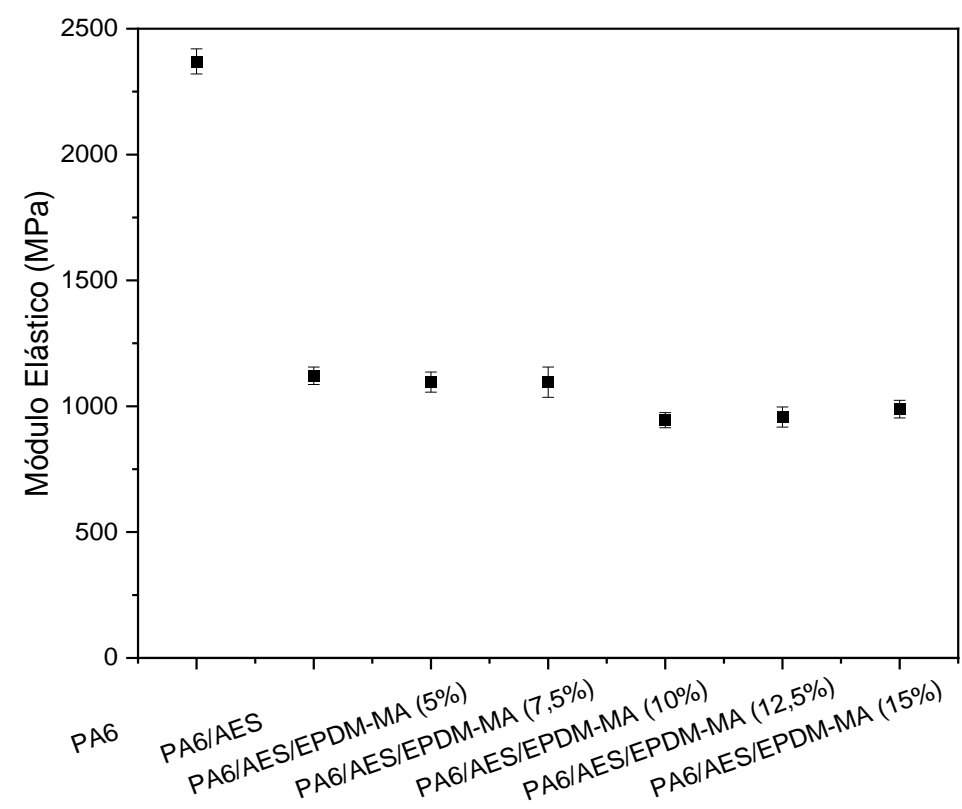

Fonte: Dados da Pesquisa. 
Na Figura 2, verificou-se que a resistência à tração atingiu um valor máximo para a PA6 pura, sugerindo que necessitou de uma maior carga para se deformar. Com a adição do AES, houve uma redução na resistência à tração, devido ao acréscimo da flexibilidade. Para as blendas compatibilizadas PA6/AES/EPDM-MA, independentemente do teor de EPDMMA, o comportamento da resistência à tração é semelhante, ou seja, não houve variações significativas nessa propriedade. Em geral, as blendas PA6/AES e PA6/AES/EPDM-MA deformam-se em tensões menores, em comparação com a PA6 pura.

Figura 2. Resistência à tração da PA6 e das blendas PA6/AES e PA6/AES/EPDM-MA.

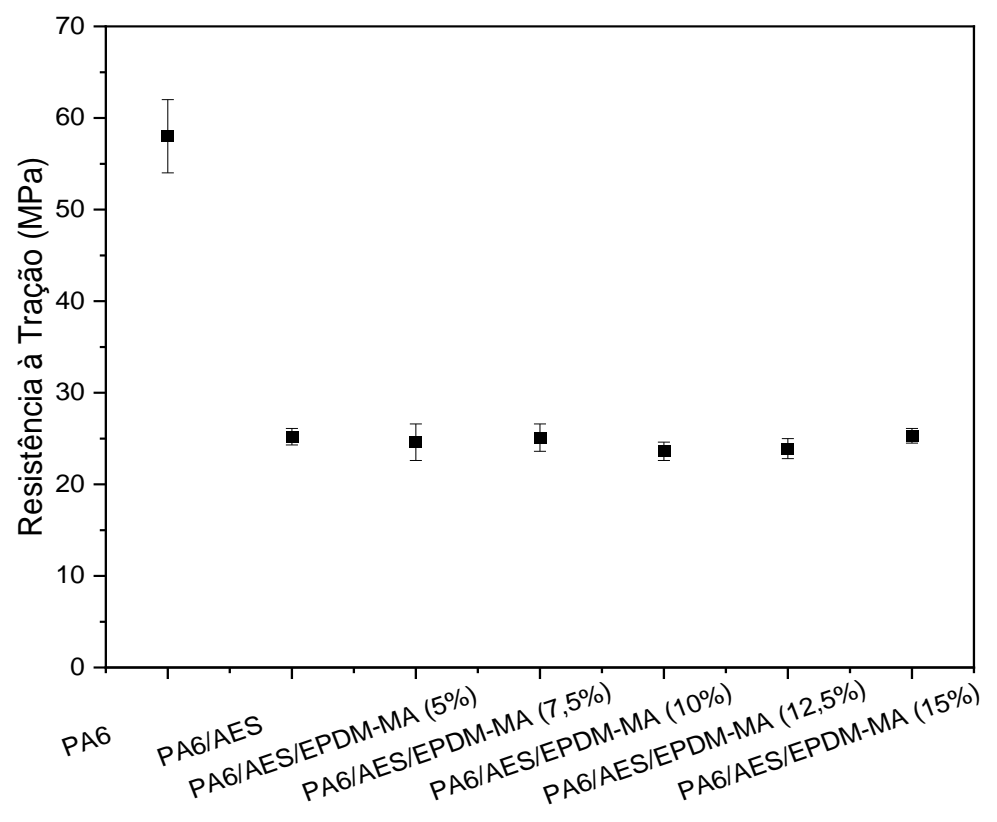

Fonte: Dados da Pesquisa.

Na Figura 3, observou-se que o alongamento na ruptura variou bastante para as blendas PA6/AES/EPDM-MA, com o aumento do teor de compatibilizante. As concentrações de EPDM-MA utilizadas aumentaram de forma contínua a propriedade de alongamento na ruptura. A adição de 15\% do EPDM-MA promoveu acréscimos na ordem de 46,5 \% e $201 \%$ no alongamento, em comparação com a PA6 e a blenda PA6/AES, respectivamente. Esse comportamento é indicativo que o EPDM-MA promoveu interações entre a PA6 e o AES, gerando um aprimoramento no mecanismo de deformação. Como o alongamento na ruptura foi uma propriedade mensurada no regime plástico, a blenda PA6/AES/EPDM-MA (15\%) aumentou o grau de ductilidade, ou seja, deforma-se mais antes da ruptura, confirmando a tendência da resistência ao impacto. 
Figura 3. Alongamento na ruptura para a poliamida 6 e as blendas.

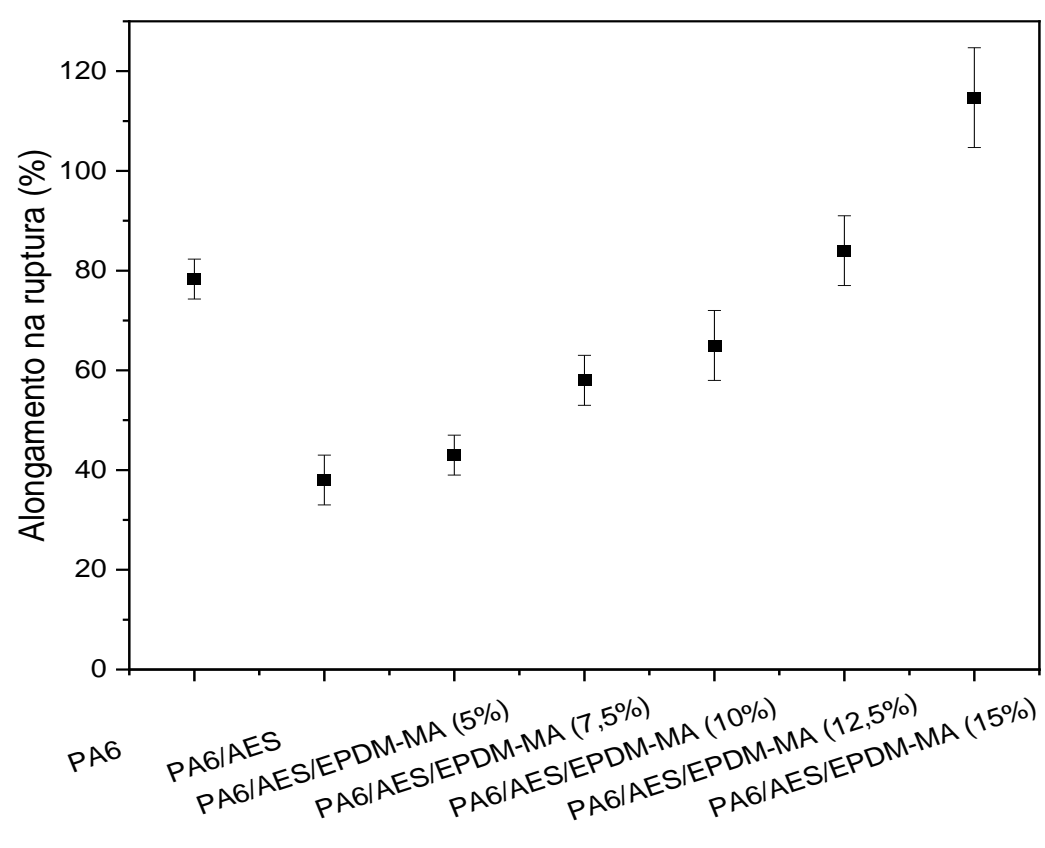

Fonte: Dados da Pesquisa.

\subsection{Comportamento mecânico sob impacto}

A Figura 4 apresenta os resultados de resistência ao impacto da PA6, da blenda PA6/AES e das misturas PA6/AES/EPDM-MA, em função do teor de EPDM-MA. A PA6 apresentou valor de resistência ao impacto na ordem de 44 $\mathrm{J} / \mathrm{m}$, indicando uma baixa capacidade de dissipação de energia quando entalhada. A blenda PA6/AES aumentou a resistência ao impacto, com ganho de aproximadamente $74 \%$ em comparação com a PA6 pura. Tal comportamento sugere que a adição individual do AES não é capaz de promover uma alta tenacificação da PA6. Quando a blenda PA6/AES é compatibilizada com EPDM-MA, independentemente do teor de compatibilizante, observou-se aumentos significativos na resistência ao impacto, em relação à não-compatibilizada (PA6/AES). Isso é indicativo que o EPDM-MA promoveu interações entre a PA6 e o AES, gerando um efeito sinérgico para aprimorar a resistência ao impacto. À medida que aumentou o teor de EPDM-MA na blenda PA6/AES/EPDM-MA, verificou-se acréscimos contínuos nessa propriedade de impacto. A blenda PA6/AES/EPDM-MA formulada com 15\% de EPDM-MA não rompeu durante o teste de impacto, com o pêndulo máximo de 5,5 J. Essa composição promoveu a formação de uma blenda supertenaz, comparável aos resultados obtidos por (Araújo et al., 2003 e Majumdar et al. 1994). Os corpos de prova da blenda PA6/AES/EPDM-MA (60/25/15\%) que não romperam estão apresentados na Figura 5. 
Figura 4. Resistência do impacto Izod para a poliamida pura, para o sistema binário e para a blenda compatibilizada.

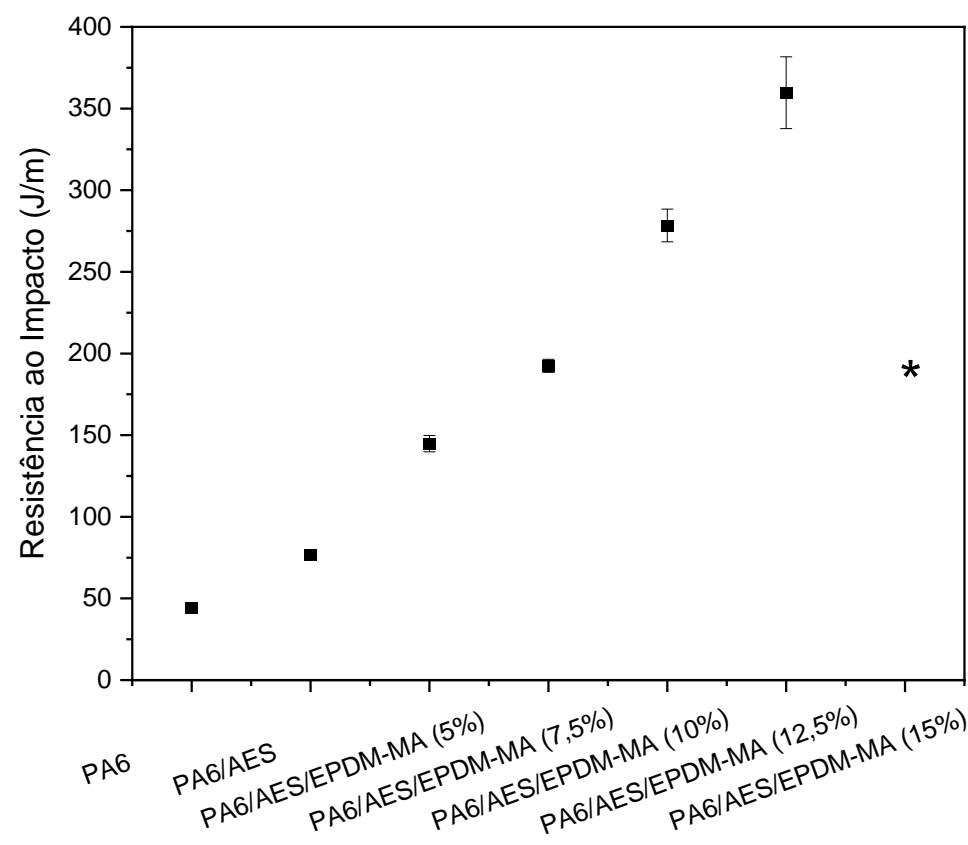

Fonte: Dados da Pesquisa.

Figura 5. Corpos de prova da blenda PA6/AES/EPDM-MA (15\%) submetidos ao teste de impacto Izod.

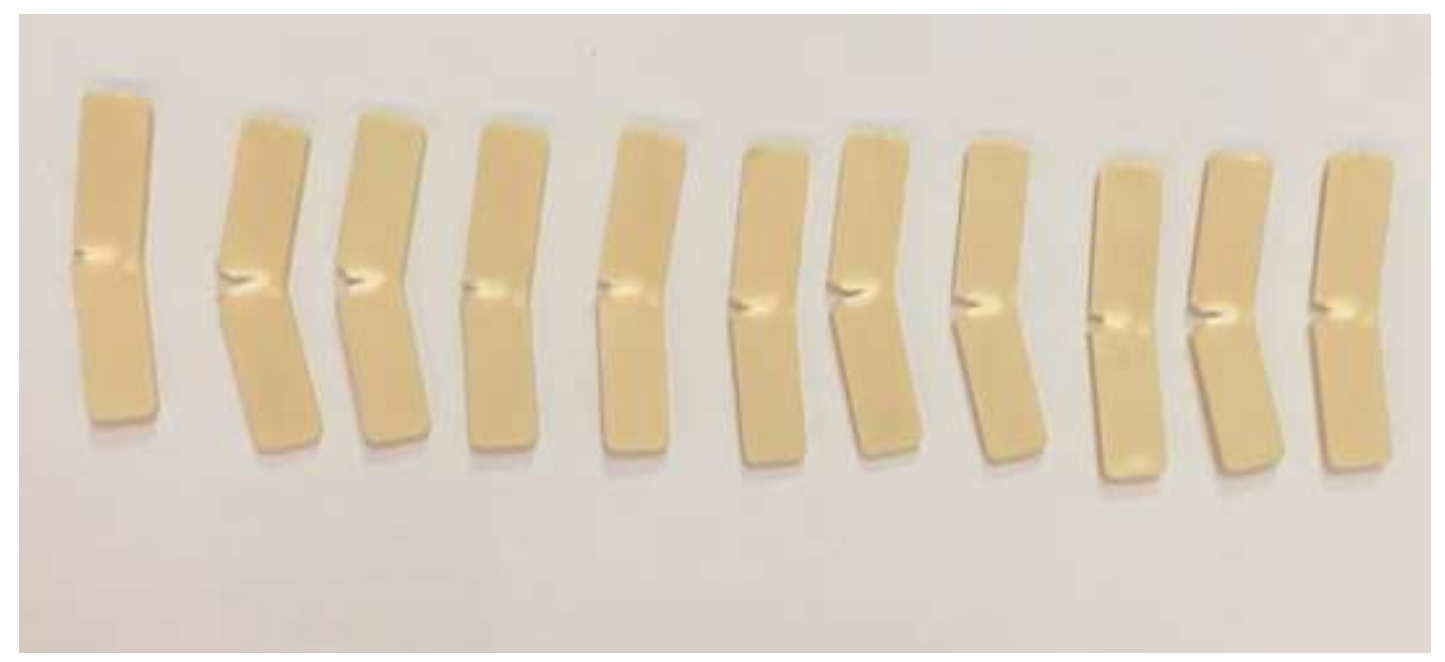

Fonte: Dados da Pesquisa.

\subsection{Microscopia Eletrônica de Varredura (MEV)}

A Figura 6 ilustra as micrografias obtidas por MEV da PA6 pura, da blenda binária PA6/AES e das blendas ternárias, com os diferentes teores de EPDM-MA. As imagens estão apresentadas com ampliação de 2000x e 5000x, respectivamente. Na Figura 6 (a), verificou-se que a PA6 pura apresentou uma morfologia com aspecto de fratura dúctil. Comportamento similar foi verificado na literatura (Silva et al., 2014). Para a blenda PA6/AES, a morfologia aumentou a rugosidade, porém a fratura foi instável devido a quantidade de partículas arrancadas durante o teste de impacto. Isso é devido à baixa interação entre a PA6 e o AES, confirmando a menor resistência ao impacto, em comparação com as blendas compatibilizadas.

As blendas PA6/AES compatibilizadas com 5 até 12,5 \% de EPDM-MA apresentaram ainda a presença de vazios, contudo com redução no diâmetro médio das partículas da fase dispersa, indicando que houve um certo grau de refinamento. 
Além disso, notou-se a presença de partículas refinadas e bem aderidas na matriz de PA6, contribuindo para tenacificar. A resistência ao impacto das blendas PA6/AES/EPDM-MA aumentou de forma expressiva, com o acréscimo de EPDM-MA. É possível que uma morfologia complexa foi desenvolvida, com partículas de AES e EPDM-MA dispersas na matriz de PA6. Ao mesmo tempo, uma fração de EPDM-MA também migrou para a interface PA6/AES, gerando um efeito sinérgico para a obtenção de altas resistência ao impacto, como verificado na Figura 5. Quando a blenda contém 15\% de EPDM-MA, o mecanismo de fratura foi diferente, em relação as blendas com menores teores de compatibilizante. Na Figura 6 (g), como não houve fratura total dos corpos de prova, a análise de MEV foi realizada na parte da superfície que flexionou durante a colisão com o pêndulo. Claramente, a fratura é de um material com alta tenacidade, devido ao alto nível de deformação plástica. Não foi possível distinguir como a fase dispersa está distribuída na matriz de PA6, tendo em vista a morfologia bastante regular e com alto nível de deformação. Aparentemente, a fase dispersa está com alto nível de adesão interfacial, contribuindo para acompanhar e aprimorar a deformação da matriz de PA6, corroborando o comportamento supertenaz.

Figura 6. Micrografias obtidas por MEV: (a) PA6 pura; (b) PA6/AES; (c) PA6/AES/EPDM-MA (5\%); (d) PA6/AES/EPDMMA (7,5\%); (e) PA6/AES/EPDM-MA (10\%); (f) PA6/AES/EPDM-MA (12,5\%); (g) PA6/AES/EPDM-MA (15\%).




Research, Society and Development, v. 10, n. 10, e210101018791, 2021

(CC BY 4.0) | ISSN 2525-3409 | DOI: http://dx.doi.org/10.33448/rsd-v10i10.18791

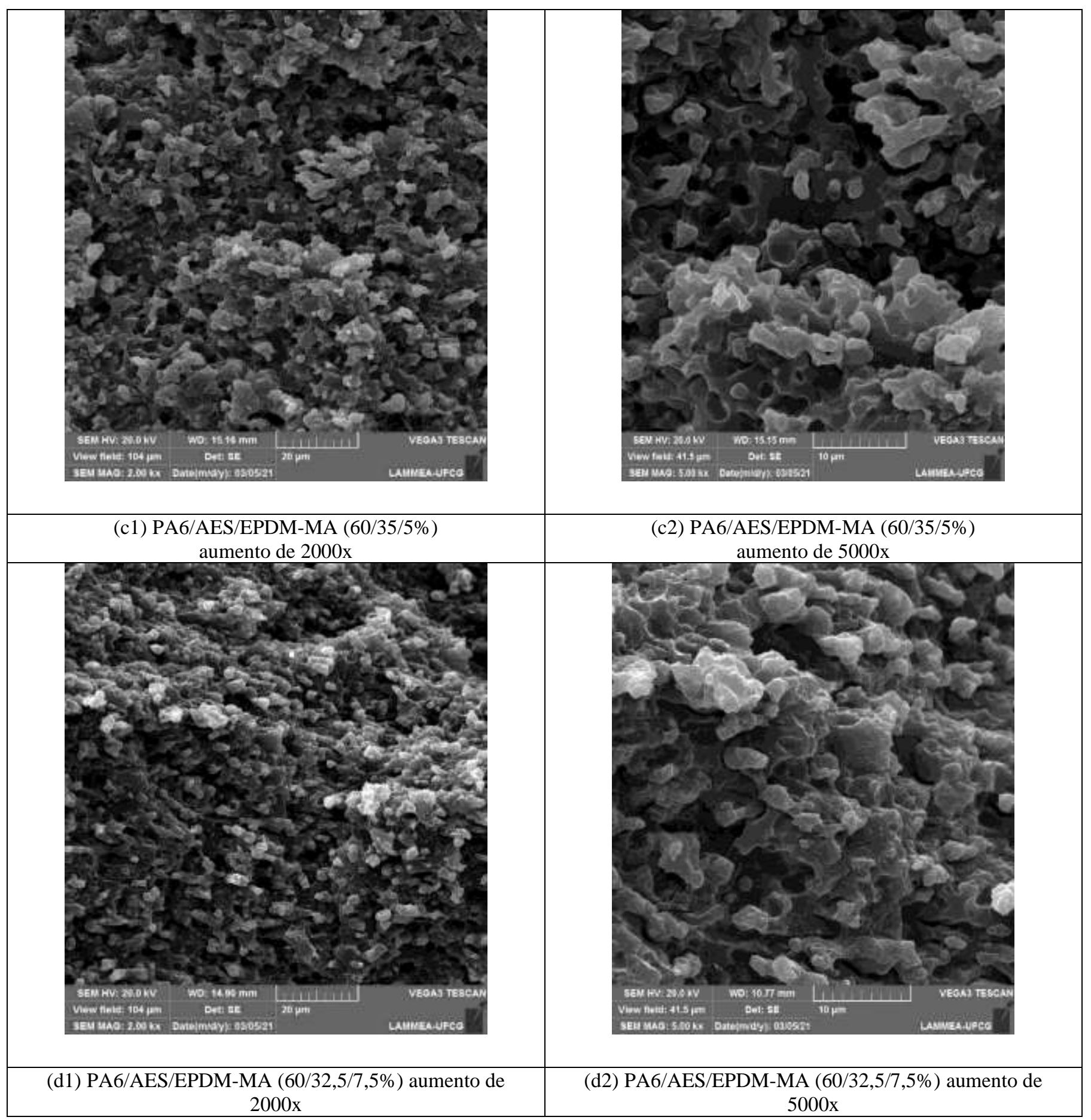


Research, Society and Development, v. 10, n. 10, e210101018791, 2021

(CC BY 4.0) | ISSN 2525-3409 | DOI: http://dx.doi.org/10.33448/rsd-v10i10.18791




Research, Society and Development, v. 10, n. 10, e210101018791, 2021

(CC BY 4.0) | ISSN 2525-3409 | DOI: http://dx.doi.org/10.33448/rsd-v10i10.18791

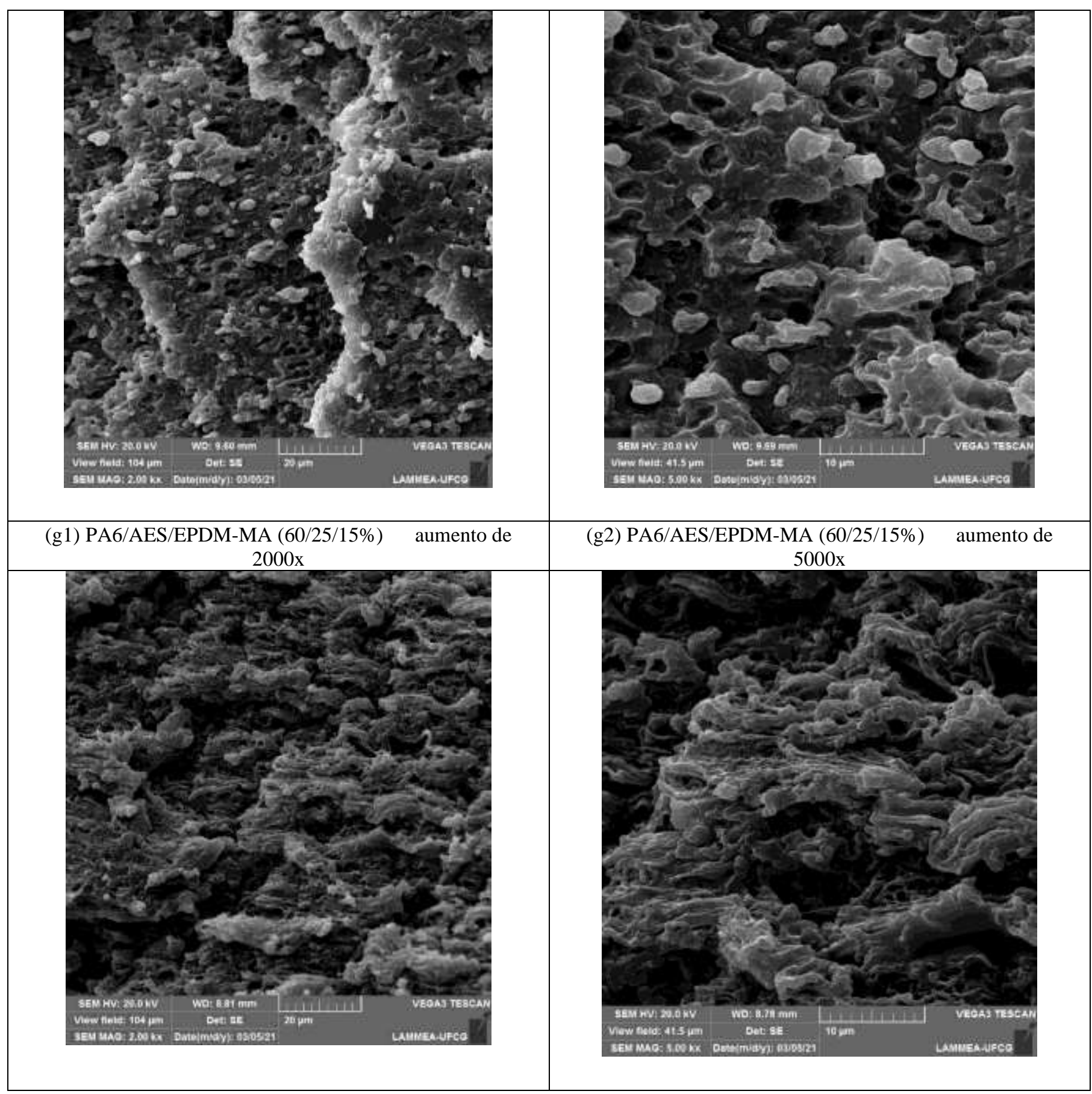

Fonte: Dados da Pesquisa.

\section{Conclusão}

Neste trabalho, o efeito da concentração do compatibilizante EPDM-MA na resistência à tração, na resistência ao impacto e na morfologia das blendas de PA6/AES foi investigado. O compatibilizante favoreceu uma maior interação entre a matriz (PA6) e a fase dispersa (AES), contribuindo para aprimorar o alongamento na ruptura e a resistência ao impacto, especialmente com 15\% de EPDM-MA. Por outro lado, houve redução no módulo elástico e na resistência à tração, indicando que as blendas se deformaram em tensões menores. Isso é atribuído ao aumento da flexibilidade das blendas, em comparação com a PA6 pura. Ficou evidenciada por meio da morfologia que o acréscimo de EPDM-MA impactou em reduzir a quantidade de vazios na blenda, bem como refinou as partículas dispersas, gerando um aumento na eficiência do mecanismo de tenacificação. A blenda PA6/AES/EPDM-MA formulada na concentração 60/25/15\% apresentou-se como um material 
supertenaz na temperatura ambiente. Essa pode ser utilizada para aplicações em que se deseja alta resistência ao impacto, como componentes da indústria automobilística.

\section{Agradecimentos}

Os autores agradecem à UFCG, à Addivant pela doação do EPDM-MA e do AES, ao MCTIC/CNPq, à CAPES/PNPD e à FAPESQ pelo apoio financeiro.

\section{Referências}

Araújo, E. M., Júnior, E. H., \& Carvalho, A. J. F. (2003). Compatibilização de Blendas de Poliamida 6/ABS usando os Copolímeros Acrílicos Reativos MMAGMA e MMA-MA. Parte 1: Comportamento Reológico e Propriedades Mecânicas das Blendas. Polímeros: Ciência e Tecnologia, $13(3), 205-211,2003$.

Bassani, A., Júnior, E. H., Pessan, L. A., Machado, A. V. \& Covas, J. (2005). A evolução da morfologia de fases de blendas PA6/AES em extrusora de dupla rosca e moldagem por injeção. Polímeros. Ciência e Tecnologia, 15(3), 176-185.

Bassani, A. Pessan, L. A. \& Júnior, E. H (2002). Propriedades mecânicas de blendas de nylon-6/ acrilonitrila-EPDM-estireno (AES) compatibilizadas com copolímero acrílico reativo (MMA-MA). Polímeros. Ciência e Tecnologia, 12(2), 102-108.

Bassani, A. Pessan, L. A. \& Júnior, E. H. (2000). Influência da composição e condições de processamento sobre o comportamento mecânico de blendas poliméricas de Nylon-6/AES. In: Congresso Brasileiro de Engenharia e Ciência dos Materiais (CBCIMAT).

Bezerra, E. B., Leite, A. M. D. \& Melo T. J. A. (2014). Preparation and characterization of membranes obtained from polymer blends of polyamide 6. Polímeros: Ciência e Tecnologia, 24(3), 381-387.

Bo, J., Feipeng, C., Xianzhong, Q., Bo, W., Guilin, J. \& Jinhua, G. (2021). The influence of extrusion process on micromorphology of PA 6/POE/POE-g-MA ternary blends: A quantitative analysis. Journal of Elastomers \& Plastics, 53(2), 110-122.

Carvalho, F. P. d., Gonçalves, M. d. C., \& Felisberti, M. I. (2010). PMMA-AES Blends Prepared by in situ Polymerization. Macromolecular Symposia, 296(1), 596-608.

Castro, L. D. C., Oliveira, A. D., Altstadt, V. \& Pessan, L. A. (2016). Effects of mixing protocol on morphology and properties of PA6/ABS blends compatibilized with MMA-MA. Journal of Applied Polymer Science, 133(27), 43612.

Essabir, H., El Mechtali, F. Z., Nekhlaoui, S., Raji, M., Bensalah, M. O., Rodrigue, D. \& Qaiss, A. (2020). Compatibilization of PA6/ABS blend by SEBS-gMA: morphological, mechanical, thermal, and rheological properties. The International Journal of Advanced Manufacturing Technology, 110(3-4), 10951111 .

Fernandes, L. L., Freitas, C. A., Demarquette, N. R. \& Fechine, G. J. M. (2012). Estudo do efeito do tipo de polipropileno na fotodegradação da blenda polipropileno/poliestireno de alto impacto. Polímeros: Ciência e Tecnologia, 22(1), 61-68.

Garcia, F. \& Lourdes, M. (1994). Sintesis y caracterizacion estructural de nuevas poliamidas com unidades monometiléncias: aplicaciones potenciales en el campo de la biomedicina. Tese Doutorado. Universidade Politécnica de Catalunya.

Ghidoni, D., Fasulo, G. C., Cecchele, D., Merlotti, M. \& Sterzi, G., (1993). A study on compatibilization of AES/PA6 blends. Journal of Materials Science, 28(15), 4119-4128.

Hage, E. \& Pessan, L. A. (2001). Aperfeiçoamento em Tecnologia de plásticos. Apostila Módulo 7: Blendas Poliméricas. Universidade Federal de São Carlos, p.142.

He, R., Zhan, X., Zhang, Q. \& Chen, F. (2017). Toughening of polyamide-6 with little loss in modulus by block copolymer containing poly(styrene-alt-maleic acid) segment. Journal of Applied Polymer Science, 134(27), 44849.

La Mantia, F. P., Morreale, M., Botta, L., Mistretta, M. C., Ceraulo \& M., Scaffaro, R. (2017). Degradation of polymer blends: A brief review. Polymer Degradation and Stability, 145(11), 79-92.

Lipatov, Y.S. \& Nesterov, A. E. (1998). Thermodynamics of Polymer Blends. Technomic Publishing, 1(1), 450.

Luna, C. B. B., Silva, D. F. \& Araújo, E. M. (2015a). Estudo do comportamento de blendas de poliamida 6/resíduo de borracha da indústria de calçados. Revista Univap, 20(36), 98-110.

Luna, C. B. B., Silva, D. F., Araújo, E. M., Mélo, T. J. A., Oliveira, A. D. (2015b). Estudo do comportamento mecânico, termomecânico e morfológico de misturas de poliestireno/composto de borracha reciclada (SBR). Matéria (Rio J.), 20 (2), 322-334.

Luna, C. B. B., Ferreira, E. S. B., Siqueira, D. D., Silva, W. A., Araújo, E. M., Wellen, R. M. R. (2019). Tailoring performance of PP/HIPS/SEBS through blending design. Materials Research Express, 6(11), 115321.

Luna, C. B. B., Siqueira, D. D., Ferreira, E. S. B., Silva, W. A., Nogueira, J. A. S. \& Araújo, E. M. (2020a). From disposal to technological potential: reuse of polypropylene waste from industrial containers as a polystyrene impact modifier. Sustainability, 12(13), 5272. 
Research, Society and Development, v. 10, n. 10, e210101018791, 2021

(CC BY 4.0) | ISSN 2525-3409 | DOI: http://dx.doi.org/10.33448/rsd-v10i10.18791

Luna, C. B. B., Siqueira, D. D., Ferreira, E. S. B., Araújo, E. M. \& Wellen, R. M. R. (2020b). Reactive compatibilization as a proper tool to improve PA6 toughness. Materials Research Express, 6(12), 125367.

Luna, C. B. B., Siqueira, D. D., Ferreira, E. S. B., Araújo, E. M. \& Wellen, R. M. R. (2021). Reactive processing of PA6/EPDM-MA blends as modifier for application and development of high-performance polypropylene. https://doi.org/10.1002/vnl.21846.

Majumdar, B., Keskkula, H., \& Paul, D. R. (1994). Effect of the nature of the polyamide on the properties and morphology of compatibilized nylon/acrylonitrile-butadiene-styrene blends. Polymer, 35, 5468-5477.

Muthuraj, R., Misra, M. \& Mohanty, A. K. (2018). Biodegradable compatibilized polymer blends for packaging applications: A literature review. Journal of Applied Polymer Science, 135(24), 45726.

Nogueira, J. A. S., Luna, C. B. B., Siqueira, D. D., Filho, E. A. S. \& Araújo, E. M. (2021). Investigação do desempenho de blendas PA6/HIPS compatibilizadas com SEBS-MA. Efeito do teor do compatibilizante nas propriedades mecânicas, termomecânicas, reometria de torque e na morfologia. Research, Society and Development, 10(8), e58510817649.

Oliveira, A. D. D., Larocca, N. M., \& Pessan, L. A. (2011). Efeito da Sequência de Mistura nas Propriedades de Blendas PA6/ABS Compatibilizadas com o Copolímero SMA. Polímeros, 21, 27-33.

Oliveira, A. D. (2014). Desenvolvimento e caracterização de nanocompósitos de blendas de pa6/aes compatibilizados com copolímero acrílico. Tese Doutorado. Universidade Federal de São Carlos.

Oliveira, A. D., Castro, L. D. C., Beatrice, C. A. G., Lucas, A. A., Pessan, L. A. (2017). Effect of Maleic Anhydride Content in Properties of PA6/AES Blends Compatibilized with MMA-MA. Materials Research, 20(6): 1630-1637.

Paul, D. R. \& Barlow, J. W. (1980). Polymer Blends. Journal of Macromolecular Science, Part C, 18(1), 109-168.

Silva, D. F., Luna, C. B. B., Araújo, E. M. \& Silva, A. L. (2016). Blendas poliméricas: conceitos, obtenção e aplicações. Revista de engenharia e tecnologia, $8(1), 58-77$.

Silva, D, F., Araújo, E. M. \& Melo, T. J. A. (2013). Development of Polyamide 6/Compound by Recycled Rubber Blends Using Graphitized Polyethylene or Polypropylene with Maleic Anhydride as Compatibilizer Agent. Journal of Aerospace Technology and Management, 5(2), 231-240.

Sridhar, A., \& Doddipatla, P. (2019). Influence of PP content on mechanical properties, water absorption, and morphology in PA6/PP blend. Journal of Applied Polymer Science, 136(25).

Turchet, R. \& Felizbert M. I. (2006) Caraterização composicional do AES - um copolímero de enxertia de poli(estireno-co-acrilonitrila) em poli(etileno-copropileno-co-dieno). Polímeros: Ciência e Tecnologia, 16(2), 157-164. 